\title{
Modelo de Simulação com Uso de Abordagem de SMA para o Zoneamento de Unidades de Conservação da Amazônia
}

\author{
Carlos Eduardo Marinelli ${ }^{1}$, Cássio Giorgio Couto Coelho ${ }^{2}$, \\ Célia Ghedini Ralha ${ }^{2}$, Alexandre Zaghetto ${ }^{2}$, Bruno Macchiavello ${ }^{2}$ \\ ${ }^{1}$ Programa Monitoramento de Áreas Protegidas - Instituto Socioambiental (ISA) \\ SCLN 210, Bloco C, Sala 112 - 70.862-530 - Brasília - DF - Brasil \\ caemarinelli@socioambiental.org.br \\ ${ }^{2}$ Departamento de Ciência da Computação - Universidade de Brasília (UnB) \\ Caixa Postal 4466 - 70.904-970 - Brasília - DF - Brasil \\ \{cassiocouto, ghedini, alexandre, bruno\}@cic.unb.br
}

\begin{abstract}
This paper presents the definition of a simulation model, with some software simulations, in order to analyze the dynamics of the occupation of farmlands within conservation units of the Amazon. The simulation model uses a multiagent system (MAS) approach based on agents' populations and the relations among them. For the agents, we have defined classes of land and farmers, in three different scenarios: preservative, intermediate and degradative. For the simulations we adopted CORMAS software, developed by the French Agricultural Research Center for International Development - CIRAD. As environments, we have used a hypothetical and a real one, based on maps of the Uacari area, Carauai district at the Amazon region.
\end{abstract}

Resumo. Este artigo apresenta a definição de um modelo de simulação, bem como a realização de simulações via software, para servir como instrumento de análise da dinâmica de ocupação de áreas agrícolas em unidades de conservação da Amazônia. O modelo de simulação utiliza a abordagem de sistema multiagente (SMA) baseado em populações de agentes e suas relações. Como agentes foram definidas classes de uso da terra/cobertura vegetal e agricultores, em três cenários diferentes: preservativo, intermediário e degradativo. Para simulação foi adotado o software CORMAS, desenvolvido pela French Agricultural Research Centre for International Development - CIRAD. Em relação aos ambientes foram utilizados um hipotético e outro real, baseado no mapa da reserva de Uacari, no município de Carauai da Amazônia legal.

\section{Introdução}

A ocupação da terra na zona de uso intensivo de unidades de conservação (UC) é uma das principais preocupações com relação ao manejo de áreas protegidas de uso sustentável na Amazônia legal. Isso porque, essa é a única classe de zoneamento onde a conversão da cobertura florestal é permitida com fins de instalação de infraestrutura física, para apoio a gestão e para o estabelecimento de comunidades humanas, além de outras atividades menos impactantes, desde que em acordo com o plano de manejo de cada UC. 
Seguindo os modos de vida tradicionais da Amazônia, uma das destinações mais comuns para o uso da terra da zona de uso intensivo de UC são aquelas voltadas para as práticas da agricultura familiar, principalmente no cultivo da mandioca. Entretanto, o manejo do uso da terra para essa finalidade também pode variar de acordo com os objetivos da produção (subsistência e/ou comercialização), e práticas tradicionais de manejo agrícola próprias de cada região da Amazônia.

Entender a dinâmica de uso da terra para fins agrícolas nas UC da Amazônia, pode contribuir para a geração de cenários mais favoráveis para manutenção da cobertura florestal, e assim, subsidiar propostas de zoneamento sob uma visão mais holística, que considere as especificidades locais, potencializando a missão das UC de uso sustentável na Amazônia legal.

Com o objetivo de fornecer suporte a tomada de decisão sobre o uso da terra e para otimizar o zoneamento de áreas protegidas, este trabalho apresenta o uso da abordagem de SMA, através de uma ferramenta de simulação que permite a análise da dinâmica de ocupação de áreas agrícolas, contribuindo para a definição de planos de gestão ambiental.

O restante do artigo será apresentado da seguinte forma: na Seção 2, apresentamos uma discussão da problemática envolvida no trabalho; na Seção 3, é detalhada a proposta de solução; na Seção 4, são apresentadas as simulações realizadas; e finalmente, na Seção 5, apresentamos as conclusões e trabalhos futuros.

\section{Discussão da Problemática Envolvida}

Cada vez mais, o conceito de manejo de ecossistemas tem sido explorado sob a ótica da complexidade [Holling 1987], sendo o uso de SMA uma das abordagens mais inovadoras que vêm sendo adotadas. Isso porque sua aplicação pode apoiar, tanto a discussão de conceitos, como o entendimento das relações entre as ciências ambientais e sociais [Bousquet and Le Page 2004]. Motivadas pela heterogeneidade de situações que envolvem as interações entre esses dois componentes distintos e complementares esta abordagem vem atraindo cada vez mais pesquisas [Bousquet et al. 2005, Bousquet et al. 2007, Barreteau et al. 2007].

Na Ciência da Computação a área de SMA lida com a autonomia dos agentes ganhando representatividade para fins de organização de sistemas constituídos por interações entre os diversos agentes de forma complexa e inteligente [Huhns and Singh 1998, Weiss 2000, Wooldridge 2002]. Trabalhos anteriores de [Balmann 1997] e [Dean et al. 2000] apresentam resultados de análises voltadas tanto ao manejo de áreas agrícolas [Deadman and Gimblett 1994], como para o manejo de áreas protegidas.

A abordagem de SMA tem contribuído nas observações sobre a conectividade de elementos ecossistêmicos, bem como em suas interações e organização em diferentes escalas. Desta forma, o uso desta abordagem pode ajudar no entendimento da dinâmica de áreas agrícolas, subsidiando a construção de propostas de zoneamento em cenários mais preservativos. Vale ressaltar que o foco da abordagem de SMA, neste caso, não está voltada para a racionalidade dos agentes, mas para as interações e ações dos mesmos através de modelos espaciais e temporais, que envolvam diferentes cenários, refletindo os elementos ecossistêmicos definidos para análise. 
Alguns dos nossos trabalhos anteriores, citam a relevância de utilizar dados científicos no auxílio para formulação de políticas de conservação na Amazônia [Mesquita and Marinelli 2007], bem como na execução de programas que envolvem a complexidade da dinâmica entre o homem amazônico e suas relações com o uso de recursos naturais em áreas protegidas [Marinelli et al. 2007].

Os dados para a definição do modelo de simulação deste trabalho são originários de uma expedição científica realizada em 2006 na Reserva de Desenvolvimento Sustentável (RDS) de Uacari, que ocupa uma área de 625.000 ha, no município de CarauariAM. Esta reserva tem uma área agrícola com cerca de 300 roçados, distribuídas em 250 ha, onde o tipo de agricultura é basicamente familiar itinerante. A expedição foi realizada pela Secretaria de Estado do Meio Ambiente e Desenvolvimento Sustentável do Amazonas (SDS), com apoio do Instituto de Proteção Ambiental do Estado do Amazonas (IPAAM), do Programa Áreas Protegidas da Amazônia (ARPA) e da Gordon and Betty Moore Foundation, no âmbito da Rede de Conservação do Amazonas. Como resultado foram identificados várias formas de utilização dos recursos naturais nesta reserva, entre eles o plantio da mandioca para produção de farinha, o qual será utilizado no modelo de simulação deste trabalho.

\section{Proposta de Solução}

Este trabalho define um modelo de interação entre agentes espacializados, envolvendo três cenários distintos, utilizado para a simulação de agentes de um SMA. Como objetivos principais do uso do modelo de simulação definido podemos citar:

- A integração de geoinformação à abordagem de SMA, como forma de suprir a carência de ferramentas que possibilitem a execução de simulações entre diferentes tipos de agentes espacializados;

- Testar o efeito de diferentes dinâmicas de uso agrícola da terra sobre a conversão de paisagens florestais (com mapas reais) e a degradação de ecossistemas;

- Analisar os padrões de resposta da cobertura vegetal para elaboração de propostas de ocupação da zona de uso intensivo de UC na Amazônia;

- Utilizar como subsídio a definição do modelo de interação entre os agentes, a realização de diversas simulações e suas respectivas análises para a definição e desenvolvimento de uma ferramenta específica de simulação, com uso de técnicas de SMA e visualização, que evidencie o comportamento de agentes espacializados inseridos em diferentes cenários ecossistêmicos.

\subsection{Ferramenta de Simulação}

A ferramenta de simulação utilizada neste trabalho foi o Common-Pool Resources and Multi-agent Systems - CORMAS, v. 3.2, (http: / / cormas . cirad.fr/en/outil/ download/). No CORMAS os diferentes tipos de controle determinam a sensitividade da simulação e as relações entre comportamentos dos grids definem a dinâmica espacial. O CORMAS foi desenvolvido na plataforma VisualWorks e linguagem Smalltalk. Os componentes básicos adotados pelo programa e que definem os agentes e suas interações podem ser divididos em três tipos: espacializados (células ou manchas), comunicativos (comunicação entre entidades) e/ou ambos (espacializado e comunicativo).

O CORMAS permitiu a geração de cenários de interação entre agentes de forma simples e rápida, sendo que as principais vantagens de aplicação é que ele prioriza as 
questões dos usos de espaços comuns em redes de interação, e cria objetos espacializados que correspondem a um grupo capaz de se comunicar e mudar sua posição neste espaço [Bousquet et al. 1998].

\subsection{Modelo de Simulação Definido}

O modelo definido inclui dois tipos de agentes distintos:

1. Classes de uso da terra/cobertura vegetal (agente espacializado), com valores médios de tamanho de $10.000 \mathrm{~m}^{2}$ (1 ha) para área de cada roçado, baseado no conhecimento empírico adquirido na expedição apresentada na Seção 2.

2. Agricultores (agentes espacializados e comunicativos).

Como de acordo com a Lei do SNUC, no máximo $5 \%$ da área total de uma UC pode ser atribuída para fins agrícolas, somente 500 (do total de 10.000 células do grid) foram consideradas como roçado no estado inicial da simulação. Logo, quando o modelo é aplicado em um ambiente hipotético, utiliza-se a conversão máxima de áreas da UC para fins agrícolas.

A lógica do comportamento dos agentes foi definido conforme o Algoritmo 1, o qual foi implementado em Smalltalk no CORMAS.

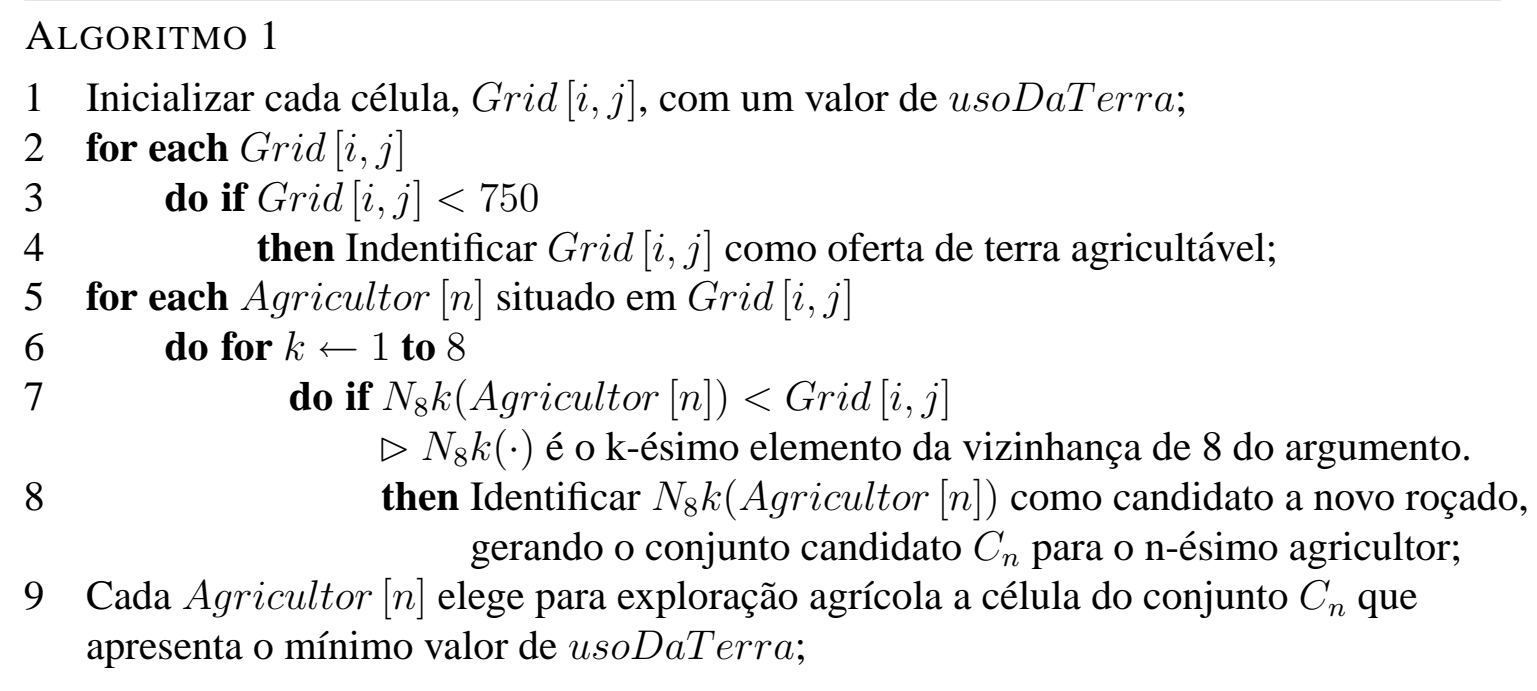

A natureza da simulação realizada é de população de agricultores, já a dinâmica de ocupação é o uso da terra em três cenários distintos, os quais representam os estados dos objetos especializados, a saber: preservativo, intermediário e degradativo. Cada cenário é estabelecido conforme três critérios principais:

- Classe de uso da terra;

- Duração do ciclo de uso da terra em cada roçado; e

- Mudança da área de plantio que determina o final de cada ciclo de uso de um roçado; considerando a redução da produtividade do solo.

O sistema de classificação dos estados dos objetos refere-se à classe de uso da terra, para os quais foram estabelecidos os seguintes estados de conservação do solo:

- Mata bruta: terreno que nunca foi utilizado para cultivo; 
- Capoeira velha: constituída pelas áreas que não são mais utilizadas para agricultura há algum tempo ou estão começando a ser utilizadas para cultivo;

- Capoeira nova: aparece logo após o abandono das áreas de agricultura (roçados) ou representan a evolução de uma capoeira velha;

- Roçado: terreno que se roçou para ser cultivado;

- Área em abandono: terreno que não pode ser mais utilizados para cultivo por serem desfavoráveis ou por necessitarem de muito tempo para se recuperar.

A partir destes estados foi determinado um tempo médio de três anos para que uma área mude de um estado para o outro. Sendo que o estado de Mata Bruta termina no momento em que a área é utilizada como área agrícola. A transição de três anos entre cada estado inclui intervalos de 250 unidades, esta divisão foi feita de tal forma a poder acompanhar visualmente a transição dos estados durante a simulação. A Figura 1 apresenta as definições do modelo para a simulação com a simbologia adotada. Vale ressaltar que o estado de área em abandono representa a não disponibilidade da terra para uso como área agrícola, ou seja, nenhum roçado poderá ser aberto nesta região, fato confirmado pelo Algoritmo 1, onde a oferta de área agricultável vai até o limite de 750.

\begin{tabular}{|l|ccc|} 
Estado & Cobertura Vegetal & Intervalo de Estado & Símbolo \\
\hline 1 & Mata bruta & 0 & $\square$ \\
2 & Capoeira velha & $1-249$ & $\square$ \\
3 & Capoeira nova & $250-499$ & $\square$ \\
4 & Roçado & $500-749$ & $\square$ \\
5 & Área em abandono & $750-1000$ & $\square$
\end{tabular}

Figura 1. Estados de conservação do solo com seus respectivos intervalos de estado e simbologia adotados na simulação.

A sequiência de estados que retrata essa configuração em cada cenário está apresentada na Figura 2. Note que trata-se de uma máquina de estados determinística, onde ao final de cada estado intermediário, isto é capoeira velha, capoeira nova e roçado, um novo ciclo de uso da terra pode recomeçar com a abertura de uma área de roçado nova e o abandono da área anteriormente utilizada.

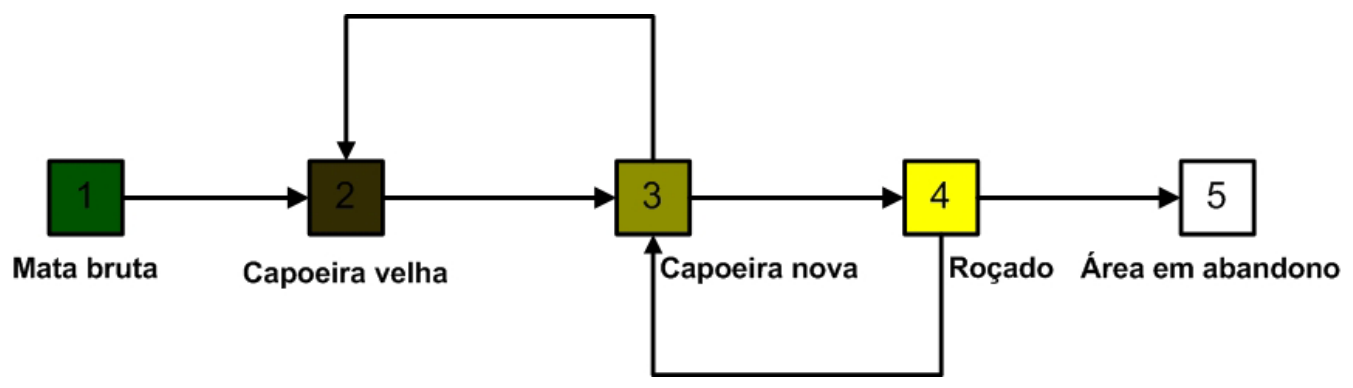

Figura 2. Máquina de estados do modelo de solução.

O modelo de simulação descrito foi aplicado em dois ambientes distintos: um hipotético e outro real, baseado no mapa da reserva de Uacari, no município de Carauai da Amazônia legal. Para uso do modelo de simulação com mapas reais, foram adotados os mesmos cenários de degradação, a mesma lógica de comportamento dos 


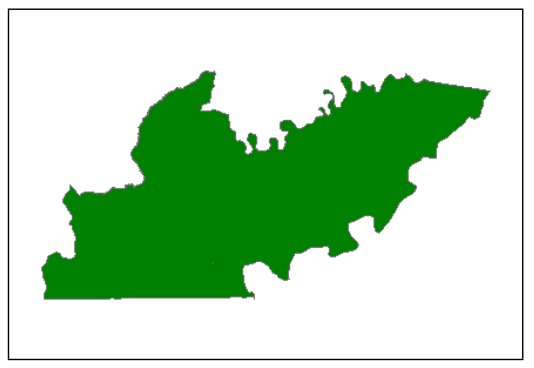

(a)

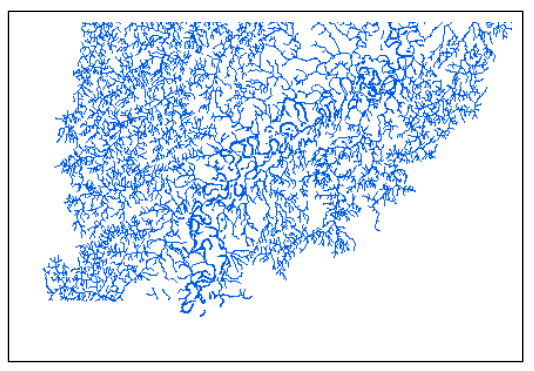

(c)

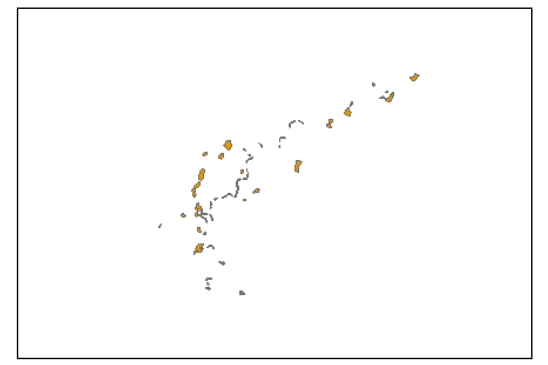

(b)

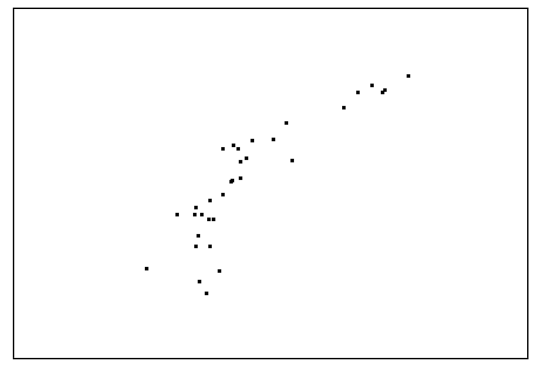

(d)

Figura 3. Shapefiles de interesse: (a) limites da região estudada; (b) roçado; (c) rios; e (d) comunidades.

agentes, com a mesma máquina de estados do ambiente hipotético. Porém, foi considerado apenas a existência de oito agentes agricultores por comunidade, que se refere a média de roçados por família (um) a partir da média de famílias por comunidade (1roçado/família $\times 8$ famílias/comunidade). Como mencionado anteriormente, no caso do ambiente hipotético, foi considerado como estado inicial a atribuição de $5 \%$ da área total para fins agrícolas.

\subsection{Integração do GIS com CORMAS}

Um dos principais objetivos do presente artigo é o uso do modelo de simulação definido para a integração de geoinformação à abordagem de SMA. Além de testar o efeito de diferentes dinâmicas de uso agrícola da terra sobre a conversão de paisagens florestais explorando a possibilidade de utilizar mapas reais, como por exemplo o mapa da RDS do Uacari. Sendo assim, o presente artigo propõe a geração de grids a partir de um Sistema Informação Geográfica ou Geographic Information System - GIS, o qual pode ser integrados ao CORMAS. A seguir será detalhado como foi feito a integração.

O primeiro passo foi identificar os shapefiles de interesse. Shapefiles são arquivos manipulados por um GIS, que contêm mapas vetoriais que descrevem elementos geográficos, sendo aqui utilizados: (a) os limites da região da RDS do Uacari; $(b)$ os roçados; $(c)$ os rios; e $(d)$ as comunidades, conforme ilustrado na Figura 3.

Em seguida o shapefile que define os limites da reserva é aberto em um editor de dados geoespacial como, por exemplo, o $\operatorname{ArcMap}^{\circledR}$, da ESRI (http: //www.esri.com/). Define-se, então, no espaço de cores RGB (Red, Green, Blue) [Gonzalez and Woods 2002], a cor da região como $R=0, G=128$ e $B=0$. O contorno da região recebe os mesmos valores RGB, e o fundo recebe a cor branca ( $R=255, G=255, B=255)$. O aspecto final do mapa é o mostrado na Figura 3 


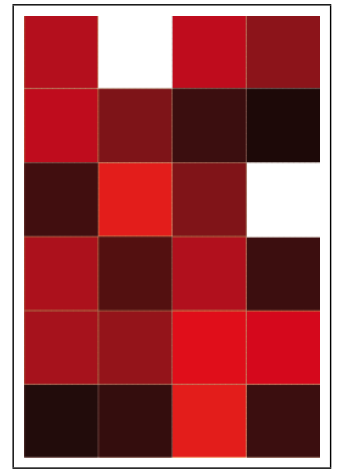

(a)

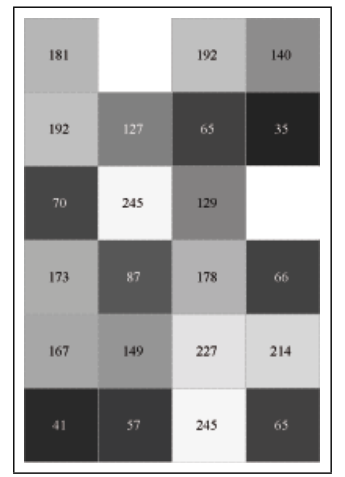

(b)

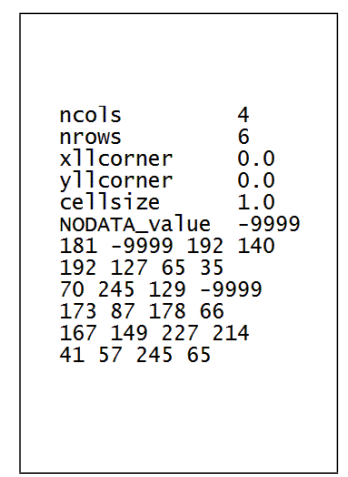

(c)

Figura 4. Conversão entre BMP e ESRI ASCII Raster: (a) imagem BMP original, no espaço de cores RGB; (b) apenas o plano R; e (c) arquivo resultante no formato ESRI ASCII Raster.

(a). Esse mapa é exportado para um formato de imagem que o editor GIS suporta. No presente artigo utilizou-se o formato BMP. É comum em editores dessa natureza permitir a definição da resolução da imagem exportada. Isso se dá por meio da escolha do valor de um parâmetro denominado DPI (dots per inch). Aqui, esse parâmetro recebe o valor 127, definido de tal forma que cada pixel represente 1 ha de área real. Como consequência, a imagem BMP exportada apresentou as dimensões de $1110 \times 1666$ pixels.

A imagem BMP é, então, convertida para o formato ESRI ASCII Raster [ESRI 2010]. Esse formato foi desenvolvido com o objetivo de possibilitar a transferência de informação entre sistemas baseados em células. Um arquivo no formato ESRI ASCII Raster começa com um cabeçalho, seguido dos valores de cada célula. É importante ressaltar, que a ferramenta de conversão aqui utilizada exporta apenas o plano $\mathrm{R}$ para o formato ASCII. A Figura 4 mostra um exemplo de: (a) uma imagem BMP representada no espaço de cores RGB; (b) apenas o plano R; e (c) o arquivo resultante no formato ESRI ASCII Raster. Os dados de cabeçalho mais relevantes, no que diz respeito a integração com o CORMAS, são ncols, nrows e NODATA_value. Os dados ncols e nrows representam o número de colunas e linhas do grid, respectivamente, e o dado NODATA_value é um valor que indica uma célula que não deve ser considerada como parte do grid.

Prosseguindo, os shapefile dos rios, dos roçados e seus contornos, e das comunidades foram imageadas sobre os limites da reserva. As cores utilizadas para estes elementos foram branco $(R=255, G=255, B=255)$, amarelo $(R=249, G=170, B=0)$ e preto ( $R=1, G=1, B=1)$, respectivamente. O tamando das comunidades foi estabelecido como um quadrado de $2 \times 2$ pixels, para facilitar a visualização. O mapa resultante foi exportado para uma imagem BMP, conforme ilustrado na Figura 6 (a), que em seguida foi convertida para o formato ESRI ASCII Raster, segundo os procedimentos anteriormente expostos. Note que as regiões de roçados, na Figura 6 (a), inclui as áreas de acesso aos mesmos, e representam na verdade um conjunto de vários roçados menores, por isso o tamanho deles graficamente representado é maior a 1 ha. O conjunto de dados do arquivo ASCII ficou assim constituído:

- 0 (verde) $\Longleftrightarrow$ Mata bruta; 
- 1 (preto) $\Longleftrightarrow$ Comunidade;

- 249 (laranja) $\Longleftrightarrow$ Roçado; e

- 255 (branco) $\Longleftrightarrow$ Rios.

Levando-se em consideração que o arquivo ASCII gerado será utilizado em uma simulação no CORMAS, é necessário compatibilizar os valores do conjunto de dados gerado com os estados expostos na Figura 1. Para efeito de simplificação vamos considerar como condição inicial da simulação que todas as áreas de roçado reais correspondem ao limite inferior do intervalo do estado "roçado", conforme definido na Figura 1. Uma outra simplificação será considerar os rios como pertencentes ao estado "área em abandono", uma vez que não são considerados para efeito de exploração agrícola. As comunidades não são consideradas agentes no sistema proposto, apenas devem servir de indicação para início da atividade dos agentes agricultores. A última etapa de pré-processamento do arquivo para importação no CORMAS é a substituição dos valores 255 para 750 , com o objetivo de caracterizar as céluas referentes aos rios e demais regiões brancas como sendo "área em abandono", os valores das comunidades, 1, para algum outro valor superior a 1000 (aqui sugerimos o valor 1001), e os valores referentes aos "roçados", 249, para 500.

\section{Simulações Realizadas}

Os resultados da simulação explicitam que as relações entre os agentes (classes de uso da terra/cobertura vegetal e agricultores) têm efeitos diferentes sobre a dinâmica de ocupação de área agrícolas. Em linhas gerais, a manutenção de uma matriz de paisagem florestal composta por mata bruta, ou sua conversão em áreas dominadas por vegetação de capoeiras (em diferentes estágios de regeneração) é um efeito resultante do tipo de manejo adotado no ciclo de rotação das áreas agricultáveis, e não do número de agricultores ou roçados.

Para cada um dos três cenários, perservativo, intermediário e degradativo, foram executados 1.000 passos/steps de simulação, conforme o modelo de simulação definido na Seção 3.2. A Figura 5 apresenta os resultados de simulações em três cenários, com grids gerados a partir de um ambiente hipotético, para intervalos de 250 passos. O grid possui uma área total de 10.000 ha, onde cada célula do grid equivale a 1 ha. Note que para melhorar a visualização na Figura 5 é mostrada apenas 100 ha da área total (ampliação).

Comparando o estado final nos três cenários, podemos perceber a perda de vegetação remanescente (mata bruta), a diminuição da distância entre os roçados e a heterogeneidade de classes ao longo de toda a simulação. Um comportamento degradativo com a quantidade máxima permitida de agentes agricultores praticamente não possui nenhuma área de mata bruta no final da simulação. O principal produto deste ciclo perverso de degradação é a formação de extensos ecossistemas de vegetação secundária, onde o desmatamento converteu a matriz originalmente florestal em extensas áreas em regeneração.

A simulação representada na Figura 5 não utiliza um mapa real, e portanto para utilização do mapa da RDS do Uacari, foi utilizada a integração do GIS com CORMAS, tal como descrito na Seção 3.3. Para a execução desta simulação foram escolhidas três seções ou grids, conforme apresentado na Figura 6. Estes três grids foram escolhidos de tal forma que somente uma comunidade esteja presente em cada um deles. O tamanho de cada grids é grande o suficiente para que não seja necessário explorar nas bordas 


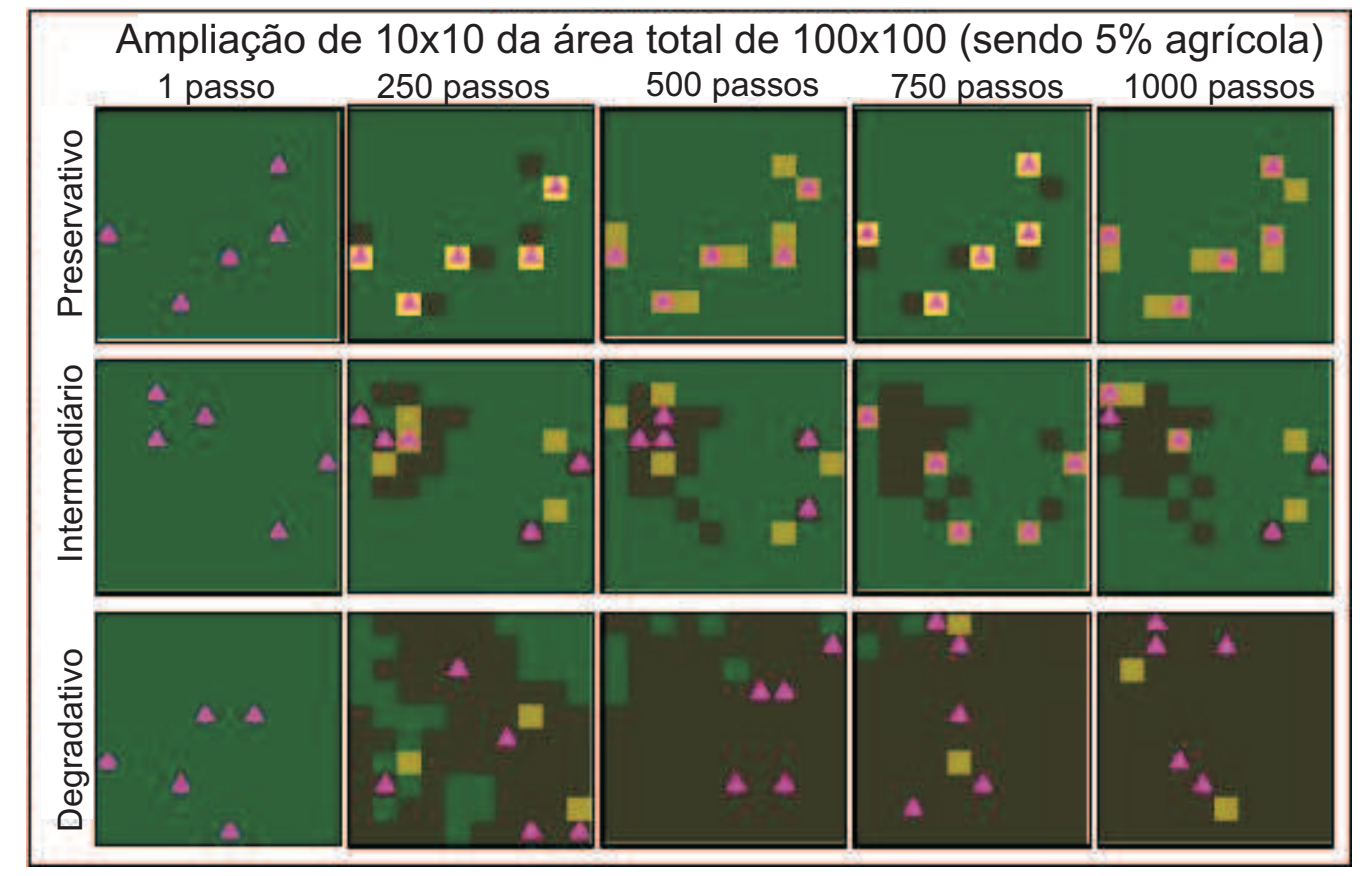

Figura 5. Simulação de três cenários em ambiente hipotético, com área total de 10.000 ha, sendo mostrados apenas 100 ha. Os agricultores são indicados por triângulos magenta. A cor de cada célula indica o seu respectivo estado, conforme a simbologia da Figura 1.

dos grids, dado que o valor médio de agricultores por comunidade nesta reserva é de 8 agricultores, como citado na Seção 3.2.

Na Figura 7 é mostrado o resultado da simulação para o grid da Figura 6 (d) com 1.000 passos de simulação. Este grid foi selecionado devido ao fato de possuir a melhor visualização das etapas da simulação. Os resultados observados para os outros dois grids, mostrados nas Figuras 6 (b) e (c), são similares aos apresentados na Figura 7. Como podemos observar existe uma grande diferença entre o comportamento preservativo e degradativo. Já o comportamento intermediário é similar ao preservativo. É importante lembrar que a quantidade de agricultores é muito inferior ao limite máximo permitido e um aumento significativo na quantidade de agricultores pode causar uma devastação da mata bruta, especialmente no comportamento degradativo, como foi observado na simulação com o ambiente hipotético da Figura 5.

\section{Conclusões e Trabalhos Futuros}

Neste trabalho propomos um modelo de simulação, para auxílio à tomada de decisão, na elaboração e revisão de planos de gestão ambiental, mas especificamente relacionados ao zoneamento de UC de uso sustentável, com estudo de caso realizado na RDS de Uacari, município de Carauari, na regial da Amazônia legal. Como principais objetivos alcançados por este trabalho podemos citar:

- Exploração de conceitos ecológicos de degradação e recuperação ambiental, para conservação da cobertura vegetal, considerando princípios do manejo integrado de ecossistemas em UC da Amazônia legal; 


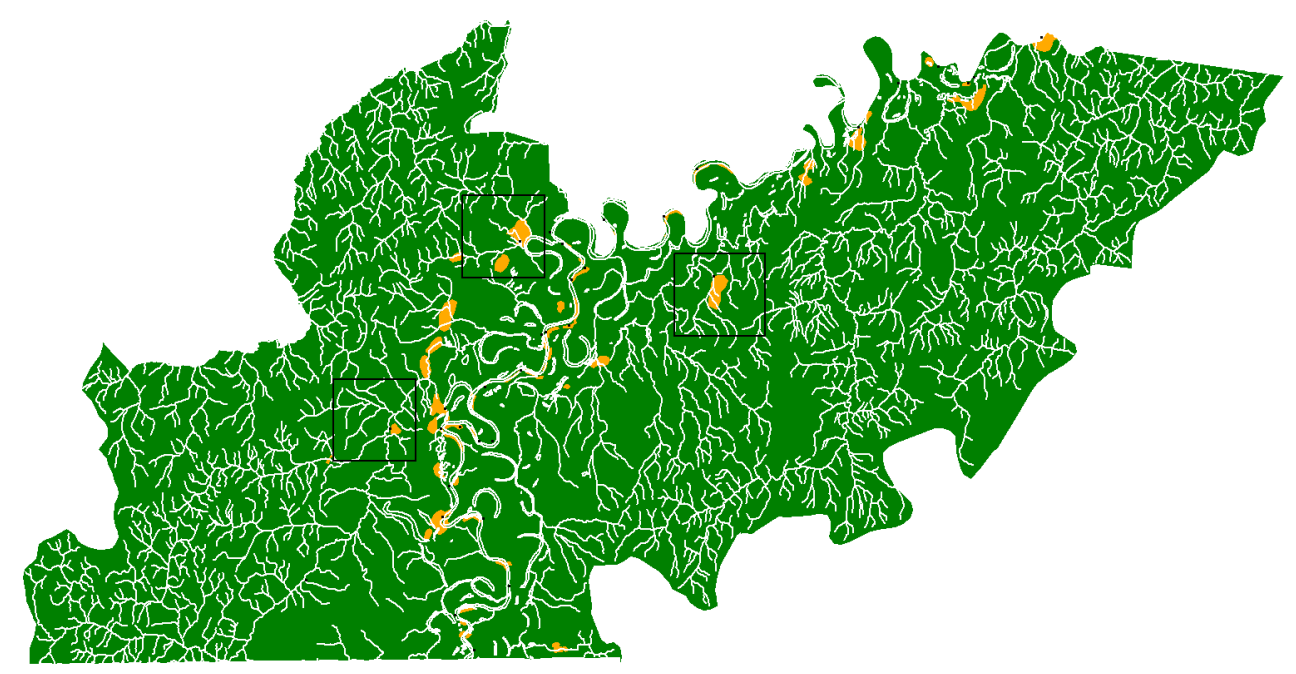

(a)

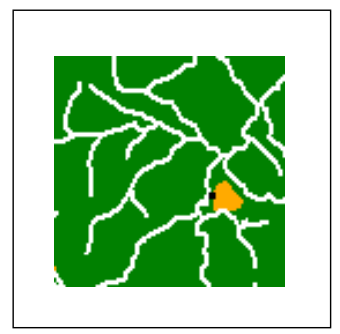

(b)

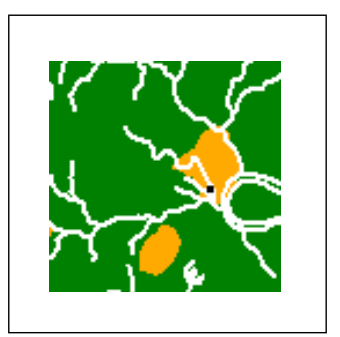

(c)

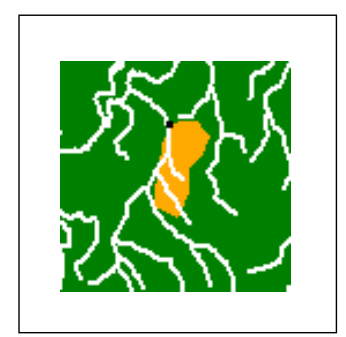

(d)

Figura 6. Trechos do mapa real da RDS de Uacari, considerados para simulação: (a) mapa resultante do imageamento dos rios, roçados e comunidades sobre os limites da RDS do Uacari. Os quadrados em destaque indicam apenas três trechos do mapa; (b), (c) e (d) são três grids distintos de $100 \times 100$ células (1 ha cada célula) considerados para simulação.

- Utilização da abordagem de SMA integrada a geoinformações, com a finalidade de definir um modelo de simulação para ser utilizado na ferramenta CORMAS;

- Teste do efeito de diferentes dinâmicas de uso agrícola da terra considerando as paisagens florestais e a degradação de ecossistemas reais através da execução de simulações do modelo definido com vários ambientes, inclusive o mapa real da RDS de Uacari;

- Análise dos padrões de resposta da cobertura vegetal para elaboração de propostas de zoneamento na RDS de Uacari;

- Subsidiar o desenvolvimento de testes experimentais, ou simulações mais complexas, envolvendo um maior número de entidades, agentes e objetos em diferentes cenários ecossitêmicos, que favoreçam cenários preservativos na UC das RDS da Amazônia.

Em prosseguimento aos trabalhos de pesquisa iniciados pela junção das abordagens de SMA à Gestão Ambiental, denominado Projeto ReasonBalance, estamos definindo uma ferramenta que utilizará SMA segundo a abordagem de Ciência da Computação. O objetivo do projeto é a possibilidade de definição de uma ferramenta de simulação com tipos diferentes de agentes e capacidades racionais distintas, os quais irão 


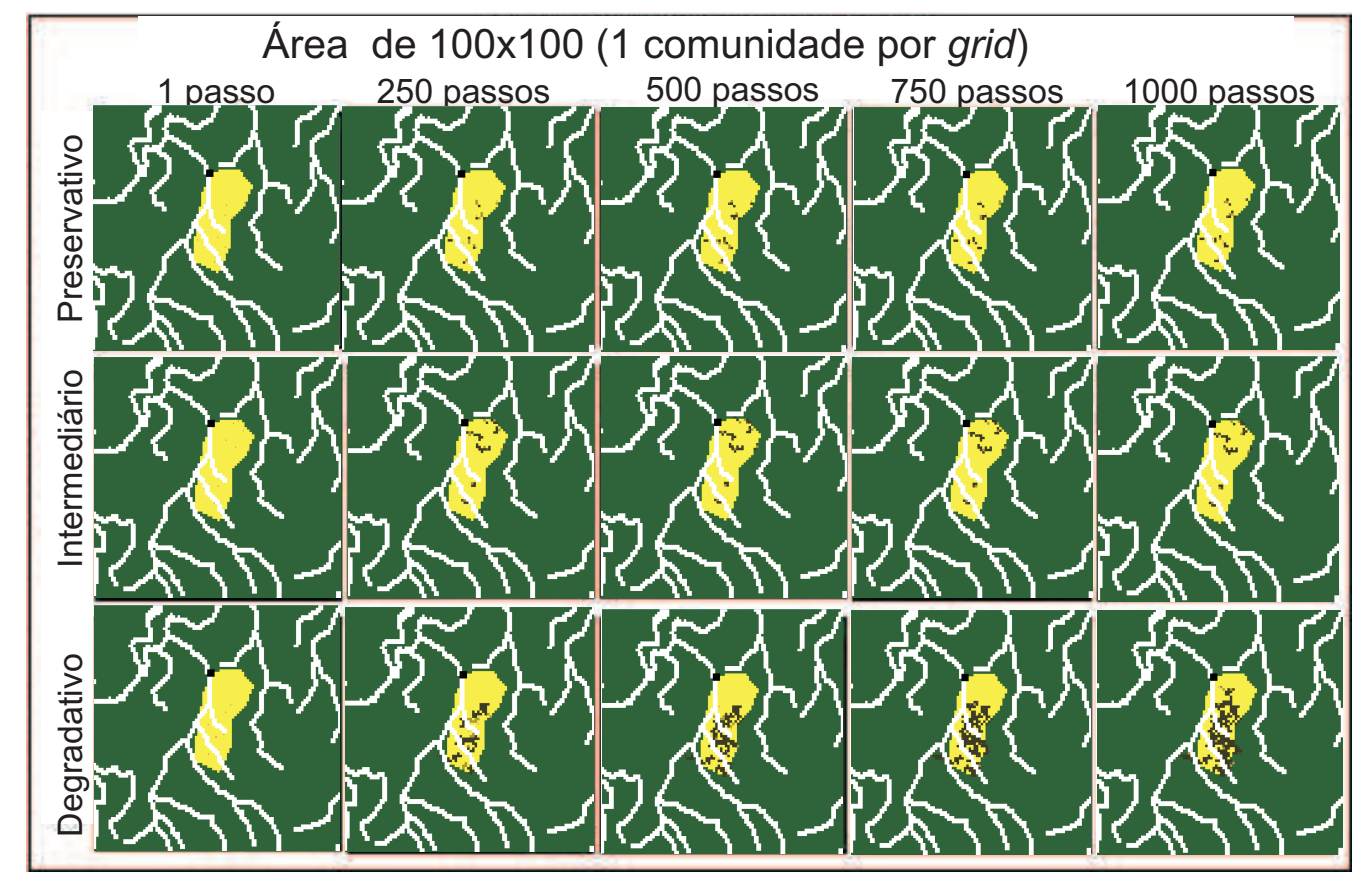

Figura 7. Simulação do grid da Figura 6 (d), na RDS de Uacari, com 1.000 passos de simulação.

interagir em diferentes níveis, através de uma definição arquitetural que permita ampliar as restrições percebidas na ferramenta de simulação CORMAS; como por exemplo, reduzir a estaticidade do ambiente de simulação com um grid mais flexível. Para esta fase pretendemos utilizar o ambiente de desenvolvimento Java Agent DEvelopment Framework $J A D E$, o qual já possui várias bibliotecas pré-definidas para auxílio ao desenvolvimento de SMA [Bellifemine et al. 2007, Italia Lab (TILAB) ].

Uma vez definidos os requisitos da ferramenta e implementado o protótipo, pretendemos fazer experimentações e evoluir no desenvolvimento de novas técnicas inteligentes a serem incorporadas ao ambiente, como o uso de algoritmo genético, para descoberta de possibilidades de cenários de simulação; e aprendizado de máquina, para melhorar a performance dos agentes em suas ações de interação nos cenários. Também na área de processamento de imagens, muitas abordagens podem ser incorporadas ao projeto de desenvolvimento da ferramenta, permitindo maior flexibilidade na visualização e integração de diferentes formatos e padrões específicos de mapas e imagens para tratamento de regiões reais.

\section{Referências}

Balmann, A. (1997). Farm-based modelling of regional structural change: A cellular automata approach. European Review of Agricultural Economics, 24(1):85-108.

Barreteau, O., Page, C. L., and Perez, P. (2007). Contribution of simulation and gaming to natural resource management issues: an introduction. Simul. Gaming, 38(2):185-194.

Bellifemine, F. L., Caire, G., and Greenwood, D. (2007). Developing Multi-Agent Systems with JADE. Wiley Series in Agent Technology, Sussex, England. ISBN 978-0-47005747-6. 
Bousquet, F., Bakam, I., Proton, H., and Le Page, C. (1998). Cormas: Common-pool resources and multi-agent systems. In IEA/AIE '98: Proceedings of the 11th International Conference on Industrial and Engineering Applications of Artificial In telligence and Expert Systems, pages 826-837, London, UK. Springer-Verlag.

Bousquet, F., Castella, J., Trébuil, G., Barnaud, C., Boissau, S., and Kam, S. P. (2007). Using multi-agent systems in a companion modelling approach for agroecosystem management in south-east asia. Outlook on Agriculture, 36(1):47-62.

Bousquet, F. and Le Page, C. (2004). Multi-agent simulations and ecosystem management: a review. Ecological Modelling, 176(3-4):211-424.

Bousquet, F., Trebuil, G., and Hardy, B., editors (2005). Companion modeling and multiagent systems for integrated natural resource management in asia. Metro Manila, Philippines : International Rice Research Institute. ISBN: 9712202089.

Deadman, P. and Gimblett, H. R. (1994). A role for goal-oriented autonomous agents in modelling people-environment interactions in forest recreation. Mathematical and Computer Modelling, 20:121-133.

Dean, J. S., Gumerman, G. J., Epstein, J. M., Axtell, R. L., Swedlund, A. C., Parker, M. T., and McCarroll, S. (2000). Understanding anasazi culture change through agent-based modeling. pages 179-205.

ESRI (2010). ESRI ASCII Raster format. http://webhelp.esri.com/ arcgisdesktop/9.2/index.cfm?TopicName=Raster_to_ASCII_ (Conversion).

Gonzalez, R. C. and Woods, R. E. (2002). Digital Image Processing. Prentice-Hall, EUA, 2nd edition.

Holling, C. S. (1987). Simplifying the complex: The paradigms of ecological function and structure. European Journal of Operational Research, 30(2):139-146.

Huhns, M. N. and Singh, M. P., editors (1998). Readings in Agents. Morgan Kaufmann, San Francico, USA. ISBN 1-55860-495-2.

Italia Lab (TILAB), T. Java Agent DEvelopment framework - JADE. Online. http: // jade.tilab.com.

Marinelli, C. E., Carlos, H. S. A., Batista, R., Rohe, F., and Waldez, F. (2007). O programa de monitoramento da biodiversidade e do uso de recursos naturais em unidades de conservação estaduais do amazonas. Áreas Protegidas da Amazônia ARPA, 1:75-78.

Mesquita, R. and Marinelli, C. E. amd Pinheiro, P. S. (2007). Quando a ciência ajuda a formulação de políticas de conservação na amazônia. In Py-Daniel, L. R., Deus, C. P., Henriques, A. L., Pimpão, D. M., and Riberiro, O. M., editors, Biodiversidade do Médio Madeira: Bases científicas para propostas de conservação, pages 239-244. Manaus: INPA.

Weiss, G., editor (2000). Multiagent Systems: a Modern Approach to Distributed Artificial Intelligence. The MIT Press, Cambridge, USA, $2^{\text {nd }}$ edition. ISBN 0-262-23203-0.

Wooldridge, M. (2002). An Introduction to MultiAgent Systems. John Wiley \& Sons, Ltd., Sussex, England. ISBN 0-471-49691-X. 